\title{
A descriptive study of solitary death in Yokohama City
}

\author{
Yasuhiro Kakiuchi ${ }^{*}$, Ryoko Nagao, Eriko Ochiai, Yu Kakimoto and Motoki Osawa
}

\begin{abstract}
Background: The solitary death rate in Japan is expected to continue increasing because of its growing superaged society and the rapid growth of home care in the country. To accurately determine the actual status of solitary deaths, we used a novel analysis method of combining vital statistics and ambulatory care information in Yokohama City.

Methods: Data of persons who died at home in 2013 were obtained from death certificate notifications. We also obtained the emergency transportation records that matched the cases of these death certificate notifications. Then, we gathered information regarding age, gender, marital status, and cause of death for the matched cases.

Results: There were 1890 "suspected unnatural deaths," in which most solitary deaths could be included, among all citizens who died at home $(n=4847)$. We were able to match 1503 of these cases with emergency transportation records. These 1503 cases were divided into two groups, "solitary death" $(n=349)$ and "un-solitary death" $(n=1154)$ according to the postmortem interval until finding (PMI-f). Pearson's $X^{2}$ tests conducted for the two groups revealed that there were significant differences regarding the proportion of persons who were elderly, unmarried, male, and had a hepatic disease and senility. A logistic regression analysis also showed that an increased likelihood of a prolonged PMI-f was associated with males and an unmarried status with hepatic diseases.
\end{abstract}

Conclusions: Unmarried, male sex, and liver diseases are independent risks for solitary death in Yokohama City.

Keywords: Solitary death, Postmortem interval until finding, Marital status, Vital statistics, Emergency transportation data

\section{Introduction}

Currently, Japan faces the serious concerns of rapid aging and a very low birthrate. The proportion of elderly people, aged 65 years or older, in the total population was approximately $27.6 \%$ in 2017 , but it is expected to increase to approximately $33 \%$ by 2030 [1]. Particularly, the number of solitary elderly households receiving little social and family support system is increasing yearly and has become a serious social problem that can even include "solitary death" [2, 3], generally defined as people dying alone and remaining undiscovered for a certain period of time.

Meanwhile, previous research has indicated that approximately $50 \%$ of Japanese citizens hope to die at home [4]. For most people, spending the last stage of life

\footnotetext{
*Correspondence: kakiuchi@yokohama-cu.ac.jp

Department of Forensic Medicine, Tokai University School of Medicine, 143

Shimokasuya, Isehara, Kanagawa 259-1193, Japan
}

in a desired location is an important element for achieving a desirable death [5]. Therefore, in line with such expectations among the public, home care is expected to become rapidly more common in the future $[6,7]$. On the other hand, the solitary death rate will simultaneously continue to increase.

However, it has been previously noted that the status of solitary deaths in Japan is difficult to fully understand through vital statistics alone [8]. This is because information regarding "households" is missing from the vital statistics data; thus, it is impossible to trace whether the deceased persons were living alone [8]. On the other hand, even for persons who were not living alone, there are a certain number of solitary deaths due to isolation within their households. Thus, it has been strongly argued that, regardless of whether a person lives alone, there are cases that should be considered as solitary

(c) The Author(s). 2019 Open Access This article is distributed under the terms of the Creative Commons Attribution 4.0 International License (http://creativecommons.org/licenses/by/4.0/), which permits unrestricted use, distribution, and reproduction in any medium, provided you give appropriate credit to the original author(s) and the source, provide a link to the Creative Commons license, and indicate if changes were made. The Creative Commons Public Domain Dedication waiver (http://creativecommons.org/publicdomain/zero/1.0/) applies to the data made available in this article, unless otherwise stated. 
deaths when enough time elapsed between the person's death and the discovery of death $[3,9]$.

In this study, we adopted the view that the time elapsed between death and discovery of death is important for understanding solitary deaths. We estimated the length of time from the time of death to the discovery of death by comparing copies of death certificate notifications, which are the source data for vital statistics, and emergency transportation records. We then classified persons who died at home into two groups (solitary and un-solitary death) based on the postmortem interval until the body was found. Subsequently, we aimed to elucidate the risk factors of solitary death.

\section{Material and methods}

\section{Data sources}

In this study, to correctly understand the status of solitary deaths, we used a methodology that differed from the conventional analyses that mainly relied on vital statistics alone. We employed an analytical method to perform comparisons and to match emergency transportation data and death certificate notifications gathered from vital statistics. More specifically, with the cooperation of the Medical Care Bureau of Yokohama City, we applied to the Ministry of Health, Labor and Welfare, for the use of death certificate notifications from vital statistic surveys, and we obtained the data of persons who died at home in 2013 in Yokohama City. With the cooperation of the Yokohama City Fire Bureau, we also obtained emergency transportation records that matched the cases from the death certificate notifications. These data are anonymous and are not linkable to specific persons. Then, we gathered information on age, gender, marital status, and cause of death for the matched cases.

\section{Statistical analysis}

The detailed version of the Vital Statistics Survey Death Forms of Yokohama citizens in 2013 was divided into "examined" and "attended" deaths, based on the presence of a postmortem certificate. In Japan, when causes of death found to be obvious disease or natural causes, the deaths were regarded as attended deaths and the death certificates were prepared by general physicians. On the other hand, when causes of death were not immediately found to be disease or natural causes, the deaths were not regarded as attended death but examined one, and the death certificates were prepared by medical examiners, along with police investigation. All postmortem examinations were performed by medical examiners in Yokohama City in 2013. Cases in which medical examiners had prepared death certificates were regarded as examined deaths, whereas cases in which general physicians had prepared the certificates were regarded as attended deaths (i.e., pure natural death). Then, we further sub-divided examined deaths into "pure unnatural deaths" (i.e., homicide, suicide, accidental deaths [e.g., falling and drowning] and deaths from unknown causes) and "suspected unnatural deaths" (i.e., deaths subjected to postmortem examination because the death was either unattended or because of another reason; these deaths were ultimately determined to have been caused by disease or natural causes).

According to a previous study [8], most solitary deaths can be included as "suspected unnatural deaths." Therefore, we obtained emergency transportation records that matched the suspected unnatural death cases (1503 of the 1890 cases) from the Yokohama City Fire Bureau. Then, those matched cases were divided into two groups, "solitary death" ( $n=349)$ and "un-solitary death," $(n=1154)$ according to the postmortem interval until finding (PMI-f). The PMI-f of the solitary death cases was 3 days or longer, while it was 2 days or shorter for the un-solitary deaths. This is because the dead body generally begins to decompose when PMI-f is 3 days or longer. In such cases, most of the general physicians in Japan refuse to prepare death certificates and the bodies are sent to the medical examiner's office. In other words, within 2 days after the death, death cases are generally dealt with by general physicians, as well as normal attended death cases, without any police investigations. In conclusion, in this study, we operationally defined "solitary death" as those cases for which PMI-f was 3 days or longer. Finally, information was gathered for both groups in terms of age, gender, marital status, and cause of death, from the detailed version of the Vital Statistics Survey Death Forms of Yokohama citizens in 2013.

A Pearson's $\chi^{2}$ test was conducted between two groups with regard to age, gender, marital status, and cause of death. Then, odds ratios (ORs) were calculated by a logistic regression to estimate the strength of association between the PMI-f and age, gender, marital status, and cause of death. PMI-f was used as binary data (3 days or longer as "long, solitary"; 2 days or shorter as "short, un-solitary") and set as the criterion variable, whereas age, gender, marital status, and cause of death were designated as the explanatory variables. The variance inflation factor (VIF) was used to check for multicollinearity. The significance level was set at $5 \%$ for all tests. SPSS (SPSS Statistics 19; IBM, Tokyo, Japan) was used for the analysis.

\section{Results}

Figure 1 displays the breakdown of the deaths among Yokohama citizens who died at home $(n=4847)$ among all citizens who died in $2013(n=31,573)$. There were 2305 attended deaths and 2542 examined deaths. When 


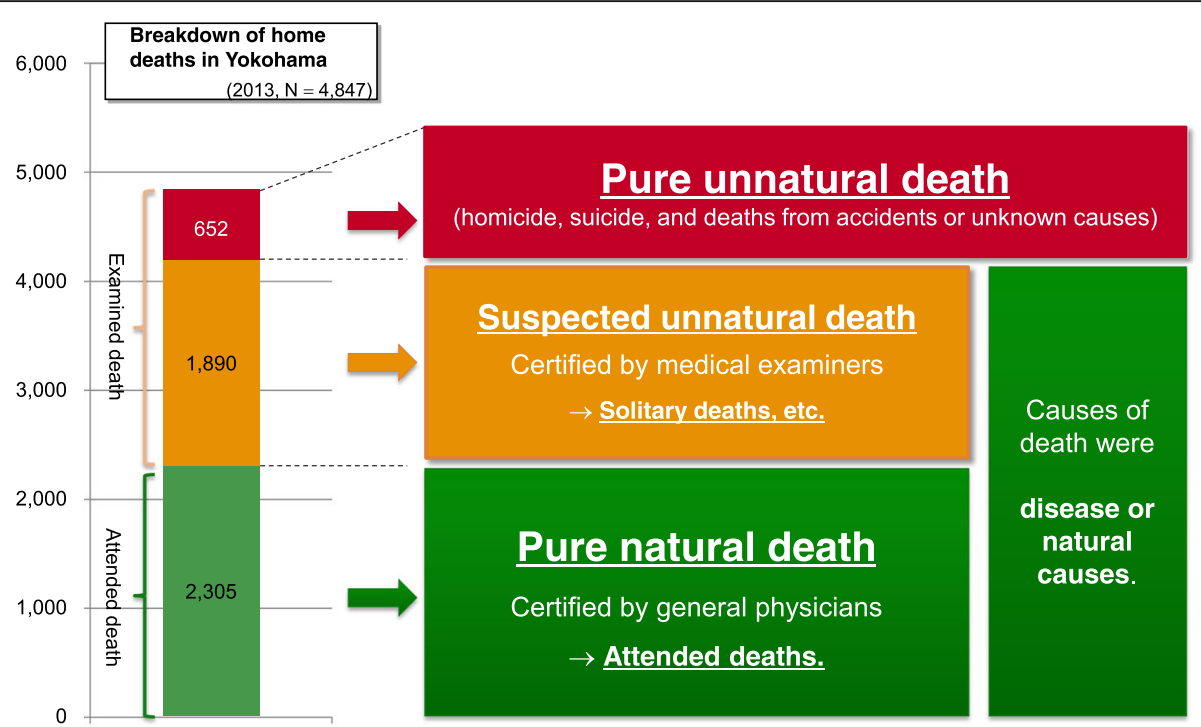

Fig. 1 Breakdown of home deaths in Yokohama City in 2013 by type of death

we further sub-divided examined deaths, we observed 652 "unnatural deaths" and 1890 "suspected unnatural deaths."

Table 1 summarizes the cases used in this study and illustrates the number of cases, proportion of elderly persons, gender, marital status, and proportion of each cause of death and $p$ value (by Pearson $\chi^{2}$ test) according to the two groups (solitary death and un-solitary death). The results of the Pearson $\chi^{2}$ test for the proportion of elderly persons, men, and unmarried persons, and autopsies among the two groups revealed that, for a long PMI-f ( 3 days or longer), the proportion of persons who were elderly $(p<0.001)$ and senile $(p=0.001)$ significantly decreased, while the proportion of unmarried persons $(p<0.001)$, men $(p<0.001)$, and persons with hepatic disease $(p<0.001)$ increased.
The logistic regression analysis results are shown in Table 2 and illustrate that all of the VIF values were below 10, indicating low multicollinearity. The odds ratios for gender (male vs. female), marital status (unmarried vs. married, divorced, and widowed), and cause of death (hepatic disease vs. all other diseases) were significant $(p<0.001$, respectively), indicating significant associations with PMI-f. However, there were no significant associations for age (youth vs. elderly, $p=0.585$ ).

\section{Discussion}

There have been many studies that have researched solitary deaths [10-18]; however, to our knowledge, this study is the first in Japan to compare the source data of death certificate notifications from the vital statistics survey with emergency transportation and to use these data

Table 1 Characteristics of study subjects

\begin{tabular}{|c|c|c|c|c|}
\hline Characteristic & & Solitary death & Un-solitary death & $p$ value \\
\hline Number of cases & & 349 & 1154 & \\
\hline Aging & Aged 65 or older (\%) & $230(65.9)$ & $912(78.9)$ & $<0.001$ \\
\hline Gender & Male (\%) & $272(77.9)$ & $691(59.9)$ & $<0.001$ \\
\hline Marital status & Unmarried (\%) & $146(41.8)$ & $193(16.7)$ & $<0.001$ \\
\hline \multirow[t]{8}{*}{ Cause of death } & Cardiovascular disease (\%) & $196(56.2)$ & $683(59.2)$ & 0.315 \\
\hline & Cerebrovascular disease (\%) & $47(13.5)$ & $138(12.0)$ & 0.452 \\
\hline & Hepatic disease (\%) & $38(10.9)$ & $45(3.9)$ & $<0.001$ \\
\hline & Respiratory disease (\%) & $27(7.7)$ & $72(6.2)$ & 0.323 \\
\hline & Malignant neoplasm (\%) & $18(5.2)$ & $61(5.3)$ & 0.925 \\
\hline & Digestive system disease (\%) & $6(1.7)$ & $23(2.0)$ & 0.744 \\
\hline & Senility (\%) & $2(0.6)$ & $52(4.5)$ & 0.001 \\
\hline & Other causes (\%) & $15(4.3)$ & $80(6.9)$ & 0.076 \\
\hline
\end{tabular}


Table 2 Factors associated with postmortem interval until finding (PMI-f) by logistic regression

\begin{tabular}{llll}
\hline Explanatory variables & OR (95\% Cl) & VIF value \\
\hline Age (youth vs. elderly (aged 65 or older)) & $1.088(0.803-1.474)$ & 1.226 & 0.585 \\
Gender (male vs. female) & $1.800(1.343-2.412)$ & 1.052 & $<0.001$ \\
Marital status (unmarried vs. married, divorced, and widowed) & $3.050(2.271-4.097)$ & 1.227 & $<0.001$ \\
Cause of death (hepatic disease vs. other diseases) & $2.475(1.547-3.960)$ & 1.014 & $<0.001$ \\
\hline
\end{tabular}

All the reference groups were the latter

$P M I-f$ postmortem interval until finding, OR odds ratio, $\mathrm{Cl}$ confidence interval, VIF variance inflation factors

in a complementary way for analysis. Moreover, one main feature of this study is that, in addition to the more common factors like age and gender, it examined marital status and cause of death, both of which had not been focused upon as much in the previous studies.

Furthermore, we attempted to understand the actual status of solitary deaths by classifying the cases in detail. As described above, we estimated the length of time from the occurrence of death to the discovery of death based on the length of time between the time of death and that of emergency transportation request. We subsequently classified "suspected unnatural death" cases into two groups according to this length of time.

The above analyses indicate that we successfully obtained a new finding regarding the risk factors for solitary death. Previous studies have reported that elderly persons living alone are at a high risk for solitary death [3]. In fact, we found that $65.9 \%$ of the cases in the solitary death group were elderly persons aged 65 years or older. In particular, the results from the Pearson's $\chi^{2}$ test demonstrate that the time from the occurrence of death to the discovery of death tended to be longer in unmarried, non-elderly males and hepatic diseases. In addition, the logistic regression analysis also showed that an increased likelihood of a prolonged PMI-f was associated with unmarried males with bad hepatic conditions. Various factors might help explain this finding. For example, according to comments in the emergency transportation records, many victims who were discovered relatively shortly after their deaths were found during periodic visits by their family members or relatives, etc. In other words, although it is true that being an elderly person living alone is certainly a risk factor for solitary death, this risk largely depends on the presence or absence of support from surrounding people who live outside the home.

Currently in Japan, the proportion of unmarried persons has been increasing annually for various reasons, including lifestyle diversification and expansion of economic disparity. Unmarried persons, when compared to their married counterparts, have a lower chance of receiving support from families and neighbors, especially when they reach middle- to elderly age. In addition, this social isolation might lead to alcohol abuse, which ultimately leads to hepatic diseases, according to the findings of previous research which stated that individual and community social isolation were associated with increased odds of drinking problems [19, 20]. This is partly because the Japanese public, in general, is culturally more tolerant to alcohol consumption than other substance abuse or smoking, etc. According to a previous research [21], more than $75 \%$ of Japanese adolescents have consumed alcohol in their first year of junior high school (when most students are aged 12 years). In the future, welfare administrative authorities should generate preventive measures against solitary death because it is possible that persons of any age living alone, especially unmarried males with hepatic diseases, can be at high risk for solitary death.

Despite the new findings described above, this study faced several limitations. Regarding the solitary death data used in this study, we collected data for only the cases that we were able to link for both the ambulatory care information system and the death certificate notifications. We could not match the remaining cases because the age or gender of the individuals in those cases was unidentified due to advanced decomposition or skeletonization of their bodies. However, there are a considerable number of solitary death cases in the data that we were unable to link. Future studies should aim to obtain the data of these latter cases to understand a more detailed status of solitary deaths. Also, because this study targeted only Yokohama City, it is uncertain whether the conclusions apply to other Japanese regions. In the future, researchers should conduct surveys that are similar to this study in other regions in order to test the validity of our conclusions. Finally, it is worth noting that the data from the ambulatory care information system, as well as the death certificate notifications, were anonymously compared. In other words, the common values between both datasets for the four items (i.e., date of onset, site of onset, age, and gender) were coupled based on pure speculation. In this study, because the quantity of data was relatively small, all data could confidently be matched one-to-one. However, for much larger sample sizes, it might be impossible to anonymously compare the datasets. To further advance studies on this issue, if necessary precautions are taken to protect personal information, breaking anonymity should be considered.

\section{Conclusion}

Unmarried, male sex, and liver diseases are independent risks for solitary death in Yokohama City. 


\section{Abbreviations}

Cl: Confidence interval; OR: Odds ratio; PMI-f: Postmortem interval until finding; VIF: Variance inflation factors

\section{Acknowledgements}

We are grateful for the support and cooperation of the Yokohama City Fire Department and Medical Care Bureau, as well as Department of Biostatistics, Yokohama City University.

\section{Funding}

This work was not supported by any foundation.

\section{Availability of data and materials}

The detailed version of the Vital Statistics Survey Death Forms of Yokohama citizens was provided from the Ministry of Health, Labour and Welfare (MHLW), in accordance with Article 33 of the Statistics Act. The personal information of the included cases was anonymized, de-identified and delivered to the Medical Care Bureau of Yokohama City. The data from the ambulatory care information system was obtained from the Yokohama City Fire Department. The data that support the findings of this study are available from the Medical Care Bureau of Yokohama City but restrictions apply to the availability of these data, which were used under license for the current study, and so are not publicly available. Data are however available from the authors upon reasonable request and with permission of the Medical Care Bureau of Yokohama City.

\section{Authors' contributions}

YaK, RN, EO, YuK, and MO made substantial contributions to the conception and design of the study, gave good analysis and interpretation of the data, and assisted in drafting the article or critically revising it for important intellectual content. YaK and $\mathrm{MO}$ made substantial contributions to the data collection and provided materials and resources. All authors read and approved the final manuscript.

\section{Ethics approval and consent to participate}

The Ethics Committee of the Tokai University School of Medicine approved the study protocol and the use of patient data (approval number: 17R-101). We also received approval from the Ethics Committee of Yokohama City Institutes of Health.

\section{Consent for publication}

Not applicable.

\section{Competing interests}

The authors declare that they have no competing interests.

\section{Publisher's Note}

Springer Nature remains neutral with regard to jurisdictional claims in published maps and institutional affiliations.

Received: 22 September 2018 Accepted: 7 February 2019

Published online: 14 February 2019

\section{References}

1. Cabinet Office, Government of Japan. Annual report on the aging society: 2017. Chapter 1 Situation on Aging. https://www8.cao.go.jp/kourei/english/ annualreport/2017/2017pdf e.htm; 2017. Accessed on 9 Jan 2019.

2. Fukukawa Y. Solitary death: a new problem of an aging society in Japan. J Am Geriatr Soc. 2011;59:174-5.

3. Nomura M, McLean S, Miyamori D, et al. Isolation and unnatural death of elderly people in the aging Japanese society. Sci Justice. 2016;56:80-3.

4. Yamagishi A, Morita T, Miyashita M, et al. Preferred place of care and place of death of the general public and cancer patients in Japan. Support Care Cancer. 2012:20:2575-82

5. Kinoshita H, Maeda I, Morita T, et al. Place of death and the differences in patient quality of death and dying and caregiver burden. J Clin Oncol. 2015; 33:357-63.

6. Akiyama A, Hanabusa H, Mikami H. Trends associated with Home Care Supporting Clinics (HCSCs) in Japan. Arch Gerontol Geriatr. 2012;54:383-6.
7. Kashiwagi M, Tamiya N, Murata M. Characteristics of visiting nurse agencies with high home death rates: a prefecture-wide study in Japan. Geriatr Gerontol Int. 2015;15:936-43.

8. Kawawaku Y, Abe N, Tanifuji T, et al. The statistics of differences in actual situation of solitary death between Tokyo 23 districts [in Japanese]. J Health Welf Stat. 2013;60:1-7.

9. Ito T, Tamiya N, Takahashi H, Yamazaki K, et al. Factors that prolong the 'postmortem interval until finding' (PMI-f) among community-dwelling elderly individuals in Japan: analysis of registration data. BMJ Open. 2012;2: e001280.

10. Gurley RJ, Lum N, Sande M, Lo B, et al. Persons found in their homes helpless or dead. N Engl J Med. 1996;334:1710-6.

11. Findlay RA. Interventions to reduce social isolation amongst older people: where is the evidence? Ageing Soc. 2003;23:647-58.

12. Cattan $\mathrm{M}$, White $\mathrm{M}$, Bond J, et al. Preventing social isolation and loneliness among older people: a systematic review of health promotion interventions. Ageing Soc. 2005;25:41-67.

13. Fujita $Y$, Inoue $K$, Seki N, et al. The need for measures to prevent "solitary deaths" after large earthquakes - based on current conditions following the Great Hanshin-Awaji Earthquake. J Forensic Legal Med. 2008;15:527-8.

14. Tadaka E, Kono A, Ito E, et al. Development of a community's self-efficacy scale for preventing social isolation among community-dwelling older people (Mimamori Scale). BMC Public Health. 2016;16:1198.

15. Luo Y, Hawkley LC, Waite $L$, et al. Loneliness, health, and mortality in old age: a national longitudinal study. Soc Sci Med. 2012;74:907-14.

16. Perissinotto CM, Cenzer IS, Covinsky KE. Loneliness in older persons: a predictor of functional decline and death. Arch Intern Med. 2012;172:1078-83.

17. Tilvis RS, Routasalo P, Karppinen $H$, et al. Social isolation, social activity and Ioneliness as survival indicators in old age: a national survey with a 7-year follow up. Eur Geriatr Med. 2012;3:18-22.

18. Dahlberg L, McKee KJ. Correlates of social and emotional loneliness in older people: evidence from an English community study. Aging Ment Health. 2014:18:504-14.

19. Murphy A, Roberts B, Kenward MG, et al. Using multi-level data to estimate the effect of social capital on hazardous alcohol consumption in the former Soviet Union. Eur J Pub Health. 2014;24:572-7.

20. Zemore SE, Ye Y, Mulia N, Martinez P, et al. Poor, persecuted, young, and alone: toward explaining the elevated risk of alcohol problems among Black and Latino men who drink. Drug Alcohol Depend. 2016;163:31-9.

21. Wada K, Price RK, Fukui S. Reflecting adult drinking culture: prevalence of alcohol use and drinking situations among Japanese junior high school students in Japan. J Stud Alcohol. 1998;59:381-6.
Ready to submit your research? Choose BMC and benefit from:

- fast, convenient online submission

- thorough peer review by experienced researchers in your field

- rapid publication on acceptance

- support for research data, including large and complex data types

- gold Open Access which fosters wider collaboration and increased citations

- maximum visibility for your research: over $100 \mathrm{M}$ website views per year

At $\mathrm{BMC}$, research is always in progress.

Learn more biomedcentral.com/submissions 